INPLASY

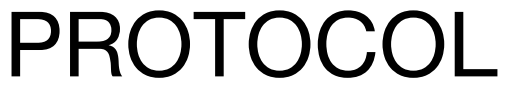

To cite: Miao et al. Effects of

Probiotics and Synbiotics

Supplementation on Insulin

Resistance in Women with

Polycystic Ovary Syndrome: A

Meta-analysis. Inplasy protocol

202150112. doi:

10.37766/inplasy2021.5.0112

Received: 31 May 2021

Published: 31 May 2021

Corresponding author:

Qin Zhang

zhaqin01@163.com

Author Affiliation:

Hangzhou TCM Hospital

Affiliated to Zhejiang Chinese

Medical University

Support: Zhejiang TCM Sci-

tech plan.

Review Stage at time of this

submission: Data analysis.

Conflicts of interest:

None declared.

\section{Effects of Probiotics and Synbiotics Supplementation on Insulin Resistance in Women with Polycystic Ovary Syndrome: A Meta-analysis}

Miao, CY1; Guo, QG²; Fang, XJ3; Chen, Y4; Zhao, Y5; Zhang, Q6.

Review question / Objective: The aim of the meta-analysis was to evaluate the effect of probiotics and synbiotics on insulin resistance in patients with PCOS.

Condition being studied: Polycystic ovarian syndrome (PCOS) is one of the most common endocrine and metabolic disorders affecting $6-26 \%$ of reproductive-aged women worldwide. The clinical manifestations of PCOS are heterogeneous and complex. In recent years, insulin resistance (IR) has been indicated as a key feature of the etiological component of PCOS, and plenty of studies have demonstrated that probiotics and synbiotics supplementation affects the metabolic status of IR. However, the effects of probiotics and synbiotics on IR among women with PCOS are controversial. Therefore, to provide accurate nutritional advice for PCOS patients, the current meta-analysis summarized the available evidence and comprehensively evaluated the effects of probiotic supplementation on the markers of IR in women with PCOS.

INPLASY registration number: This protocol was registered with the International Platform of Registered Systematic Review and Meta-Analysis Protocols (INPLASY) on 31 May 2021 and was last updated on 31 May 2021 (registration number INPLASY202150112).

\section{INTRODUCTION}

Review question / Objective: The aim of the meta-analysis was to evaluate the effect of probiotics and synbiotics on insulin resistance in patients with PCOS.
Condition being studied: Polycystic ovarian syndrome (PCOS) is one of the most common endocrine and metabolic disorders affecting $6-26 \%$ of reproductiveaged women worldwide. The clinical manifestations of PCOS are heterogeneous and complex. In recent years, insulin 
resistance (IR) has been indicated as a key feature of the etiological component of PCOS, and plenty of studies have demonstrated that probiotics and synbiotics supplementation affects the metabolic status of IR. However, the effects of probiotics and synbiotics on IR among women with PCOS are controversial. Therefore, to provide accurate nutritional advice for PCOS patients, the current meta-analysis summarized the available evidence and comprehensively evaluated the effects of probiotic supplementation on the markers of IR in women with PCOS.

\section{METHODS}

Search strategy: In the current study, we systematically searched the PubMed (https://www.ncbi.nIm.nih.gov/pubmed/), Web of Science (http://webofscience.com), Embase (https://www.elsevier.com/ solutions/embase-biomedical-research) and China national knowledge infrastructure (https://www.cnki.net/) databases through October 30, 2020, using the keywords 1) polycystic ovary syndrome or polycystic ovary disease or PCOS and 2) probiotics, prebiotics, or synbiotics. Subsequently, manual searches of the references were performed to identify possible additional studies.

Participant or population: Enrolled women with a standard diagnosis of PCOS according to the European Society of Human Reproduction and Embryology (ESHRE), the American Society of Reproductive Medicine (ASRM), or the National Institutes of Health (NIH).

Intervention: Administrated probiotic and/ or symbiotic supplementation as interventions.

Comparator: Placebo was the comparator of our study.

Study designs to be included: Randomized controlled trials (RCTs) evaluating the effects of probiotics and/or synbiotics supplementation in the treatment of patients with PCOS.
Eligibility criteria: We included studies meeting the following criteria: 1) Types of studies: randomized controlled trials (RCTs) evaluating the effects of probiotics and/or synbiotics supplementation in the treatment of patients with PCOS. 2) Types of participants: studies that enrolled women with a standard diagnosis of PCOS according to the European Society of Human Reproduction and Embryology (ESHRE), the American Society of Reproductive Medicine (ASRM), or the National Institutes of Health (NIH). 3) Types of interventions: administrated probiotic and/or symbiotic supplementation as interventions, studies administering metformin as a co-intervention in both the intervention and control groups also considered. 4) Types of outcomes: the main outcomes included HOMA-IR, serum insulin, fasting blood sugar (FBS), body mass index (BMI), waist circumference (WC), hip circumference (HC). The exclusion criteria were: studies including patients with other diseases such as Cushing's syndrome, type 1 or 2 diabetes, hyperthyroidism, or other hormone-related disorders and studies with unavailable data and unreported target outcomes.

Information sources: The information sources of our study included electronic databases and contact with authors. Published and grey literatures were searched in several databases, such as pubmed, web of science, embase and opengrey.

Main outcome(s): The main outcomes included HOMA-IR, serum insulin, fasting blood sugar (FBS), body mass index (BMI), waist circumference (WC), hip circumference (HC).

Quality assessment / Risk of bias analysis: Two authors independently assessed the quality of the included studies. The evaluation was based on the following criteria: study design and case characteristic matching, patient inclusion and exclusion criteria, and Jadad RCT guidelines (randomization, hidden allocation, blinding, and follow-up). 
Strategy of data synthesis: We carried out statistical analysis using the Review Manager software. Since we detected substantial statistical heterogeneity, we used random-effects meta-analysis to produce an overall summary of an average treatment effect across trials.

Subgroup analysis: We investigated substantial heterogeneity on the primary outcomes by using subgroup analyses, only if more than four trials reported that outcome. We had intended to do this for the two main comparisons, Comparison 1 (probiotics or synbiotics) and Comparison 2 (8-weeks intervention period or 12-weeks intervention period). However, due to the limited number of studies, we did not perform subgroup analysis.

Sensitivity analysis: We conducted a sensitivity analysis based on the quality of the studies. We considered a study to be of high quality if it was assessed as having low risk of bias in both the randomisation and allocation concealment and additionally a low risk of bias in either blinding or losses to follow-up. Conversely, we considered a study to be low quality if it was assessed as having high risk of selection bias in both the randomisation and allocation concealment. The studies that were not classified as high or low quality were classified as unclear quality.

Language: The language was limited to Chinese and English.

Country(ies) involved: China.

Keywords: Probiotics. Synbiotics. Insulin resistance. Central obesity. Polycystic ovary syndrome.

Contributions of each author:

Author 1 - Chenyun Miao.

Email: moofang560@163.com

Author 2 - Qingge Guo.

Author 3 - Xiaojie Fang.

Author 4 - Yun Chen.

Author 5 - Ying Zhao.

Author 6 - Qin Zhang.

Email: zhaqin01@163.com 